\title{
Modelos de crescimento microbiano ruminal em função do suprimento de nutrientes
}

\author{
Models of ruminal microbial growth as a function of nutrient supply
}

\author{
OLIVEIRA, André Soares de ${ }^{1 *}$; LANA, Rogério de Paula ${ }^{2}$; OLIVEIRA, Márcia \\ Rodrigues Carvalho ${ }^{3}$
}

\footnotetext{
${ }^{1}$ Universidade Federal de Mato Grosso, Instituto de Ciências Agrárias e Ambientais, Sinop, Mato Grosso, Brasil.

${ }^{2}$ Universidade Federal de Viçosa, Centro de Ciências Agrárias, Departamento de Zootecnia, Viçosa, Minas Gerais, Brasil.

${ }^{3}$ Universidade Federal de Mato Grosso, Instituto de Ciências da Saúde, Sinop, Mato Grosso, Brasil.

*Endereço para correspondência: andresoli@ufmt.br
}

\section{RESUMO}

Avaliaram-se diferentes modelos lineares (quadrático, linear-platô, quadrático-platô e raiz quadrada) e não lineares (exponencial-Mitscherlich e Michaelis-Menten) para descrever o crescimento microbiano ruminal em função do suprimento de nutrientes. Utilizou-se banco de dados compostos por 76 observações de meios de cultivo ruminal in vitro (mix de bactéria) contendo 0,$0 ; 0,375 ; 0,75$; 1,$50 ; 3,0 ; 6,0 ; 12$ e $24 \mathrm{~g}$ de sacarose/L. O crescimento microbiano foi avaliado por meio de mensuração da densidade óptica (OD - 600nm) após $0 ; 6 ; 12 ; 18$ e 24 horas de fermentação. A avaliação conjunta dos parâmetros da regressão linear dos valores preditos e observados indicou adequação de todos os modelos na predição do crescimento microbiano. A partição do quadrado médio do erro de predição não permitiu identificar diferenças na qualidade da predição do crescimento microbiano entre os modelos. Porém, ao avaliar o crescimento marginal em função do suprimento de glicose verificou-se inadequação do modelo linear-platô e do modelo quadrático (em baixos níveis substratos). Os modelos quadrático-platô, raiz quadrada, Mitscherlich ou Michaelis-Menten podem ser utilizados para descrever o comportamento do crescimento microbiano ruminal ao suprimento de nutrientes. Entretanto, diferenças são observadas quanto às estimativas das eficiências de uso nos diferentes níveis de substratos, o que implica em diferenças nas recomendações dos níveis ótimos de nutrientes. Neste sentido, a escolha desses modelos poderá ser modulada pelo nível de suprimento de nutrientes desejado.

Palavras-chave: cinética de saturação, Michaelis-Menten, resposta marginal

\section{SUMMARY}

Linear (linear-plateau, quadratic, quadraticplateau, square root) and non-linear (exponential and Michaelis-Menten) models to describe the ruminal microbial growth as a function of sucrose levels were evaluated. Is was used a database of 76 observations of microbial growth in roll tubes (mix of bacterial) containing final concentrations of $0.0 ; 0.375$; $0.75 ; 1.50 ; 3.0 ; 6.0 ; 12$ and $24 \mathrm{~g} / \mathrm{L}$ of sucrose. The microbial growth was evaluated by measuring optical density (OD - 600nm) at $0 ; 6$; $12 ; 18$ and 24 hours of fermentation. The evaluation of linear regression parameters of predicted and observed values indicated adequacy of all models in the prediction of microbial growth. The partition of the mean square prediction error did not allow identifying differences in relation to prediction quality of the models. However, the marginal growth as a function of sucrose supply was inadequate to models linear-plateau and quadratic (in low sucrose levels). The square root, Mitscherlich and Michaelis-Menten models can be used to describe biological responses to nutrients. However, differences are observed in estimates of substrates efficiencies, which implies differences in the recommendations of optimum levels of nutrients. In this sense, the choice of these models can be modulated by the level of nutrient supply desired.

Keywords: marginal response, MichaelisMenten, saturation kinetics 


\section{INTRODUÇÃO}

O conhecimento das respostas dos sistemas biológicos ao suprimento de nutrientes constitui a base fundamental para avaliações de eficiência de uso dos mesmos, bem como para elaboração de critérios de recomendações dos níveis ótimos (OLIVEIRA et al., 2010). Essa prerrogativa apresenta aplicações principalmente em sistemas de produção animal e agrícola, os quais utilizam de forma intensiva recursos escassos. Neste contexto, a escolha de modelos que melhor represente o comportamento ao suprimento de nutrientes faz-se necessário.

Os sistemas microbiológicos apresentam comportamento curvilíneo ao suprimento de nutrientes à semelhança dos sistemas macrobiológicos vegetal e animal, os quais, por sua vez, constituem essencialmente o mesmo comportamento dos sistemas enzimáticos ao suprimento de substratos (LOPEZ et al., 2004; LANA et al., 2005; MESQUITA \& NERES, 2008; OLIVEIRA et al. 2010). Tal característica apresenta implicações práticas, pois possibilita a adoção de modelos microbianos para descrever $\mathrm{o}$ comportamento da maioria dos sistemas biológicos de interesse econômico.

No entanto, a escolha do modelo matemático apresenta-se como um desafio (POUILLOT \& LUBRAN, 2011). Modelos lineares e não lineares têm sido utilizados para descrever respostas animais e vegetais ao suprimento de nutrientes, tais como linear-platô, quadrático, quadrático-platô, raiz quadrada, exponencial e MichaelisMenten (CERRATO \& BLACKMER. 1990; LANA et al., 2005; OLIVEIRA et al., 2010). Na maioria dos trabalhos, recomendações para o uso desses modelos baseiam-se em avaliações do coeficiente de determinação, o que não garante acurácia e precisão em sua predição. Altos valores de coeficiente de determinação indicam que os modelos se ajustam bem aos dados utilizados para estimá-los, mas representam pouco valor sobre a qualidade da predição dos mesmos.

Segundo os princípios da modelagem e simulação, para que um modelo seja aceito e aplicado, deverá cumprir etapas que envolvam desde a definição do problema até sua avaliação, mediante o uso de ferramentas adequadas para avaliação da qualidade de predição (TEDESCHI, 2006; OLIVEIRA et al., 2010).

Objetivou-se avaliar diferentes modelos lineares e não-lineares para descrever o crescimento microbiano ruminal ao suprimento de nutrientes.

\section{MATERIAL E MÉTODOS}

Para a construção e avaliação dos modelos, utilizou-se um banco de dados formado por 76 observações obtidas de um ensaio in vitro de crescimento microbiano ruminal ao suprimento de sacarose, descritos por Lana et al. (2007). O inóculo ruminal foi coletado no conteúdo ruminal formado por mix de bactérias. Os autores avaliaram o crescimento microbiano em tubos de ensaio que continham concentrações finais de 0,$0 ; 0,375 ; 0,75 ; 1,50 ; 3,0 ; 6,0$; 12 e $24 \mathrm{~g} / \mathrm{L}$ de sacarose. O crescimento microbiano foi avaliado mediante determinação da densidade óptica (DO $600 \mathrm{~nm}$ ), medidos nos tempos $6 ; 12 ; 18$ e 24 horas de fermentação.

Do total das observações, aproximadamente $60 \%$ foram utilizados para construção dos modelos e $40 \%$ para avaliação, as quais foram sorteadas ao acaso. No entanto, foi feita restrição na casualização quanto ao tempo de 
fermentação, de maneira a permitir distribuição igualitária dos tempos de fermentação nos dados utilizados para construção e avaliação dos modelos.

Os modelos de predição do crescimento microbiano (DO - 600nm) ao suprimento de sacarose (SAC, g/L) foram construídos baseados nas inter-relações derivadas diretamente das observações sobre $\mathrm{o}$ sistema (modelo empírico). Seis modelos de respostas foram utilizados: linearplatô, quadrático, quadrático-platô, raiz quadrada, exponencial e MichaelisMenten.

A função linear-platô seguiu o seguinte modelo:

$\mathrm{Y}=a+b \mathrm{X}$, se $\mathrm{X}<\mathrm{C}$

$\mathrm{Y}=$ platô, se $\mathrm{X} \geq \mathrm{C}$

Em que $\mathrm{Y}=$ crescimento microbiano (DO - 600nm), $X=$ concentração de sacarose $(\mathrm{g} / \mathrm{L}), \mathrm{a}=$ intercepto, $\mathrm{b}=$ coeficiente linear, $\mathrm{C}=$ concentração de sacarose máxima $(\mathrm{g} / \mathrm{L})$.

A função quadrática seguiu o seguinte modelo:

$\mathrm{Y}=a+b \mathrm{X}+c \mathrm{X}^{2}$

Em que $\mathrm{Y}=$ crescimento microbiano (DO

- 600nm), $\mathrm{X}=$ concentração de sacarose

$(\mathrm{g} / \mathrm{L}), \mathrm{a}=$ intercepto, $\mathrm{b}=$ coeficiente linear, $\mathrm{c}=$ coeficiente quadrático,

A função quadrática-platô seguiu $\mathrm{o}$ seguinte modelo:

$\mathrm{Y}=a+b \mathrm{X}+c \mathrm{X}^{2}$, se $\mathrm{X}<\mathrm{C}$

$\mathrm{Y}=$ platô, se $\mathrm{X} \geq \mathrm{C}$

Em que $\mathrm{Y}=$ crescimento microbiano (DO $-600 \mathrm{~nm}), X=$ concentração de sacarose $(\mathrm{g} / \mathrm{L}), \mathrm{a}=$ intercepto, $\mathrm{b}=$ coeficiente linear, $\mathrm{c}=$ coeficiente quadrático, $\mathrm{C}=$ concentração de sacarose máxima $(\mathrm{g} / \mathrm{L})$.

A função raiz quadrada seguiu $o$ seguinte modelo:

$\mathrm{Y}=a+b \mathrm{X}+c \mathrm{X}^{0,5}$

Em que $\mathrm{Y}=$ crescimento microbiano (DO $-600 \mathrm{~nm}), X=$ concentração de sacarose $(\mathrm{g} / \mathrm{L}), \quad \mathrm{a}=$ intercepto, $\mathrm{b}=$ coeficiente linear, $\mathrm{c}=$ coeficiente raiz quadrado.
A função exponencial de Mitscherlich seguiu o seguinte modelo:

$\mathrm{Y}=\mathrm{M}\left(1-\exp ^{-\mathrm{c}(\mathrm{X}+\mathrm{b})}\right)$

Em que $\mathrm{Y}=$ crescimento microbiano (DO

- 600nm), $\mathrm{M}=$ máximo crescimento microbiano, $\mathrm{X}=$ concentração de sacarose $(\mathrm{g} / \mathrm{L})$, c e b $=$ constante obtidas pelo ajustamento do modelos aos dados.

A função de Michaelis-Menten seguiu o seguinte modelo:

$\mathrm{Y}=\left(\mathrm{Y}_{\max } * \mathrm{X}\right) /\left(\mathrm{X}_{50 \% \max }+\mathrm{X}\right)$

Em que $\mathrm{Y}=$ crescimento microbiano (DO $-600 \mathrm{~nm}), X=$ concentração de sacarose $(\mathrm{g} / \mathrm{L}), \mathrm{Y}_{\max }=$ crescimento microbiano máximo teórico e $\mathrm{X}_{50 \% \max }=$ concentração de sacarose que permite $50 \%$ do crescimento máximo teórico.

$\mathrm{Y}_{\max }$ e $\mathrm{X}_{50 \% \max }$ foram estimados a partir do procedimento de LineweverBurk, mediante seguinte modelo:

$1 / \mathrm{Y}=\mathrm{a}+\mathrm{b} .(1 / \mathrm{X})$

Em que $\mathrm{Y}=$ crescimento microbiano (DO $-600 \mathrm{~nm}), \mathrm{X}=$ concentração de sacarose $(\mathrm{g} / \mathrm{L}), \mathrm{a}=$ intercepto $\mathrm{e} \mathrm{b}=$ coeficiente linear. Assim, $\mathrm{Y}_{\max }=1 / \mathrm{a}$ e $\mathrm{X}_{50 \% \max }=\mathrm{b} / \mathrm{a}$.

A validação dos modelos foi baseada pela relação dos valores observados e aqueles preditos, através de ajustamento de regressão linear simples dos valores preditos sobre os observados. As estimativas dos parâmetros da regressão foram testadas por intermédio do teste $\mathrm{F}$, conforme sugerido por Mayer et al. (1994), sob as seguintes hipóteses:

Ho: $\beta o=0$ e $\beta_{1}=1$

$\mathrm{Ha}$ : não $\mathrm{Ho}$

Somente sob o caso de não-rejeição de $H$ o, concluirá que o modelo prediz com similaridade os valores observados. Em todos os procedimentos estáticos adotouse 0,05 como nível de probabilidade para o erro tipo I.

A identificação da eficiência de predição dos modelos foi feita pela avaliação e decomposição do quadrado médio do erro de predição (QMEP) em quadrado do vício (QV), magnitude de 
Rev. Bras. Saúde Prod. Anim., Salvador, v.13, n.2, p....-... abr./jun., 2012 http://www.rbspa.ufba.br ISSN 15199940

flutuação (MaF) e modelo de flutuação (MoF) conforme as equações (Kobayashi \& Salam, 2000):

$\mathrm{QMEP}=\mathrm{QV}+\mathrm{MaF}+\mathrm{MoF}$

$\mathrm{QV}=(\mathrm{X}-\mathrm{Y})^{2}$

$\mathrm{MaF}=(\mathrm{SDx}-\mathrm{SDy})$

$\mathrm{MoF}=2 . \mathrm{SDx} \cdot \mathrm{SDy} \cdot(1-\mathrm{r})$

O QV representa o quadrado da diferença entre os valores preditos (X) e aqueles observados (Y). Quanto maior o QV, maior é a inacurácia dos modelos. A MaF representa a diferença entre o desvio padrão os valores preditos (SDx) e o desvio padrão dos valores observados (SDy). Quanto maior a MaF maior é a magnitude da flutuação (dispersão) entre a predição e a observação. MoF representa a correlação ponderada pelo desvio padrão entre a predição e a observação. Assim, altos valores de MoF indicam que o modelo não é adequado em simular o padrão de flutuação através das mensurações, ou seja, apresenta baixa correlação com a realidade (KOBAYASHI \& SALAM, 2000).

Para os modelos válidos $(\mathrm{P}<0,05)$ estimouse o crescimento microbiano marginal (CMa) em função da concentração de sacarose, a concentração de sacarose que permite $50 \%$ do crescimento microbiano máximo $\left(\mathrm{SAC}_{50 \% \mathrm{MAX}}, \mathrm{g} / \mathrm{L}\right)$, o crescimento microbiano máximo $\left(\mathrm{DO}_{\mathrm{MAX}}\right)$ e a concentração de sacarose no ponto de $\mathrm{DO}_{\mathrm{MAX}}\left(\mathrm{SAC}_{\mathrm{MAX}}\right)$. CMa foi estimado a partir da primeira derivada das equações. Nos modelos (3) e (6) a $\mathrm{SAC}_{\mathrm{MAX}}$ foi estimada igualando-se a zero a primeira derivada e, posteriormente, estimado DO MAX. Nos modelos (1) e (4) DO MAX $_{\text {e }}$ $\mathrm{SAC}_{\mathrm{MAX}}$ foram estimados pelo modelo (platô). No modelo (7) $\mathrm{DO}_{\mathrm{MAX}}=99,9 \%$ do crescimento máximo (M). No modelo (8) $\mathrm{DO}_{\text {Max }}$ e $\mathrm{SAC}_{50 \% \mathrm{MAX}}$ foram estimados pelo modelo Linewever-burk, descritos anteriormente. Em todos os modelos, exceto (8), $\mathrm{SAC}_{50 \% \mathrm{MAX}}$ foi obtido por procedimento iterativo.

\section{RESULTADOS E DISCUSSÃO}

Observou-se pelo comportamento da amplitude e distribuição que, as variáveis permitiram projeções e avaliações representativas de crescimento microbiano ruminal ao suprimento de nutrientes (Tabela 1). Todos os modelos ajustaram-se $(\mathrm{P}<0,05)$ ao comportamento dos dados, mas com divergências quanto ao coeficiente de determinação $\left(r^{2}\right)$ (Tabela 2). Os modelos linear-platô e Mitscherlich apresentaram maiores valores de $\mathrm{r}^{2}$ devido ao melhor ajustamento nos menores níveis de sacarose (Figura 1). No entanto, o ajustamento dos modelos aos dados não garante, por si, qualidade de predição, sendo necessárias avaliações da acurácia e precisão na predição.

A avaliação conjunta do intercepto e coeficiente de inclinação da equação de regressão dos valores observados sobre os preditos pelos modelos (Tabela 3) apontou a não-rejeição da hipótese de nulidade $(\mathrm{P}>0,05)$ para todos os modelos avaliados. Isto indica que os mesmos podem ser aplicados na predição do crescimento microbiano em função do suprimento de substratos. Desta forma, permite-se utilizá-los para estimar índices de eficiência, bem como recomendar níveis ótimos de suprimentos de nutrientes. De maneira geral, os modelos predizem com baixa presença de vício constante (viés), com valores para DO $(600 \mathrm{~nm})$ representando apenas 0,43 ; 2,$40 ; 2,37 ; 2,06$ e $4,92 \%$ do valor de DO (600nm) observado, para os modelos quadrático, linear-platô, quadrático-platô, raiz quadrada e Michaelis-Menten (Figura 2). O modelo Mitscherlich apresentou maior vício constante, representando $9,04 \%$ do valor observado para DO $(600 \mathrm{~nm})$.

No modelo quadrático, embora estime com menor vício constante entre os modelos (QV) e simule o mesmo 
Rev. Bras. Saúde Prod. Anim., Salvador, v.13, n.2, p....-... abr./jun., 2012 http://www.rbspa.ufba.br ISSN 15199940

impacto (baixo MaF), o mesmo apresenta elevada falha na direção da predição (alta participação do $\mathrm{MoF}$ ). Este comportamento pode ser melhor visualizado na Figura 3, na qual observa-se elevada dispersão dos dados acima e abaixo da reta de equalidade $(\mathrm{Y}=\mathrm{X})$. Desta forma, apresenta-se como o modelo de menor qualidade de predição do comportamento do crescimento microbiano ao suprimento de nutrientes.

Tabela 1. Estatísticas descritivas para concentração de sacarose (SAC, g/L) e crescimento microbiano ruminal medido pela densidade óptica $-600 \mathrm{~nm}$ (DO - 600nm) no conjunto de dados, para estimação dos parâmetros dos modelos e avaliação dos mesmos

\begin{tabular}{|c|c|c|c|c|c|c|}
\hline \multirow{2}{*}{ Estatísticas } & \multicolumn{2}{|c|}{ Conjunto de dados } & \multicolumn{2}{|c|}{ Estimação de parâmetros } & \multicolumn{2}{|c|}{ Avaliação } \\
\hline & SAC & DO & $\mathrm{SAC}$ & DO & SAC & DO \\
\hline Média & 5,07 & 0,59 & 4,87 & 0,57 & 5,37 & 0,61 \\
\hline Moda & 0,00 & 0,46 & 0,00 & 0,46 & 0,00 & não detectado \\
\hline Mediana & 1,50 & 0,59 & 0,75 & 0,52 & 1,50 & 0,65 \\
\hline Mínimo & 0,00 & 0,13 & 0,00 & 0,00 & 0,00 & 0,00 \\
\hline Máximo & 24,00 & 1,07 & 24,00 & 24,00 & 24,00 & 24,00 \\
\hline Desvio-padrão & 7,48 & 0,26 & 7,66 & 0,26 & 7,31 & 0,26 \\
\hline $\mathrm{n}$ & \multicolumn{2}{|c|}{76} & \multicolumn{2}{|c|}{45} & \multicolumn{2}{|r|}{31} \\
\hline
\end{tabular}

Tabela 2. Estimativas dos parâmetros, coeficiente de determinação e nível de probabilidade para o erro tipo I dos modelos de predição do crescimento microbiano ruminal medido pela densidade óptica $(\mathrm{Y}=\mathrm{DO}-600 \mathrm{~nm}) \mathrm{em}$ função da concentração de sacarose (SAC, g/L)

\begin{tabular}{llcc}
\hline Modelo & Estimativa dos parâmetros da regressão & $\mathrm{R}^{2}$ & Valor $P$ \\
\hline Linear Platô & $\begin{array}{l}0,2545+0,302 * \mathrm{SAC}, \text { platô } \mathrm{DO}=0,8133, \\
\mathrm{SAC}=1,8482\end{array}$ & 0,957 & 0,0177 \\
Quadrático & $0,382338+0,090258 * \mathrm{SAC}-0,00313 * \mathrm{SAC}^{2}$ & 0,6081 & $<0,0001$ \\
Quadrático & $0,2328+0,4244 * \mathrm{SAC}-0,07943 * \mathrm{SAC}^{2}$, & 0,8375 & $<0,0001$ \\
Platô & platô $\mathrm{DO}=0,8209, \mathrm{SAC}=2,67 \mathrm{~g} / \mathrm{L}$ & 0,7765 & $<0,0001$ \\
Raiz quadrada & $0,206035-0,0613 * \mathrm{SAC}+0,413554 * \mathrm{SAC}^{0,5}$ & 0,9627 & $<0,0001$ \\
Mitscherlich & $0,8262 *\left(1-\mathrm{exp}^{-0,8713 *(\mathrm{SAC}+0,3631)}\right)$ & 0,6114 & 0,0002 \\
Michaelis- $_{\text {Menten }}{ }^{1}$ & $(0,7882 * \mathrm{SAC}) /(0,3758+\mathrm{SAC})$ & & \\
\hline
\end{tabular}

${ }^{1}$ As constantes de Michaelis-Menten foram estimadas a partir dos coeficientes obtidos pelo modelo de transformação de modelo de Linewever-burk $(1 / \mathrm{DO})=1,2687+0,4768 \times(1 / \mathrm{SAC}), \mathrm{R}^{2}=0,6114$, onde: $\operatorname{DOmax}=1 / 1,2687=0,7882 ; \mathrm{SAC}_{50 \% \operatorname{Max}}=0,4768 / 1,2687=0,3758$. 
Rev. Bras. Saúde Prod. Anim., Salvador, v.13, n.2, p....-... abr./jun., 2012 http://www.rbspa.ufba.br ISSN 15199940
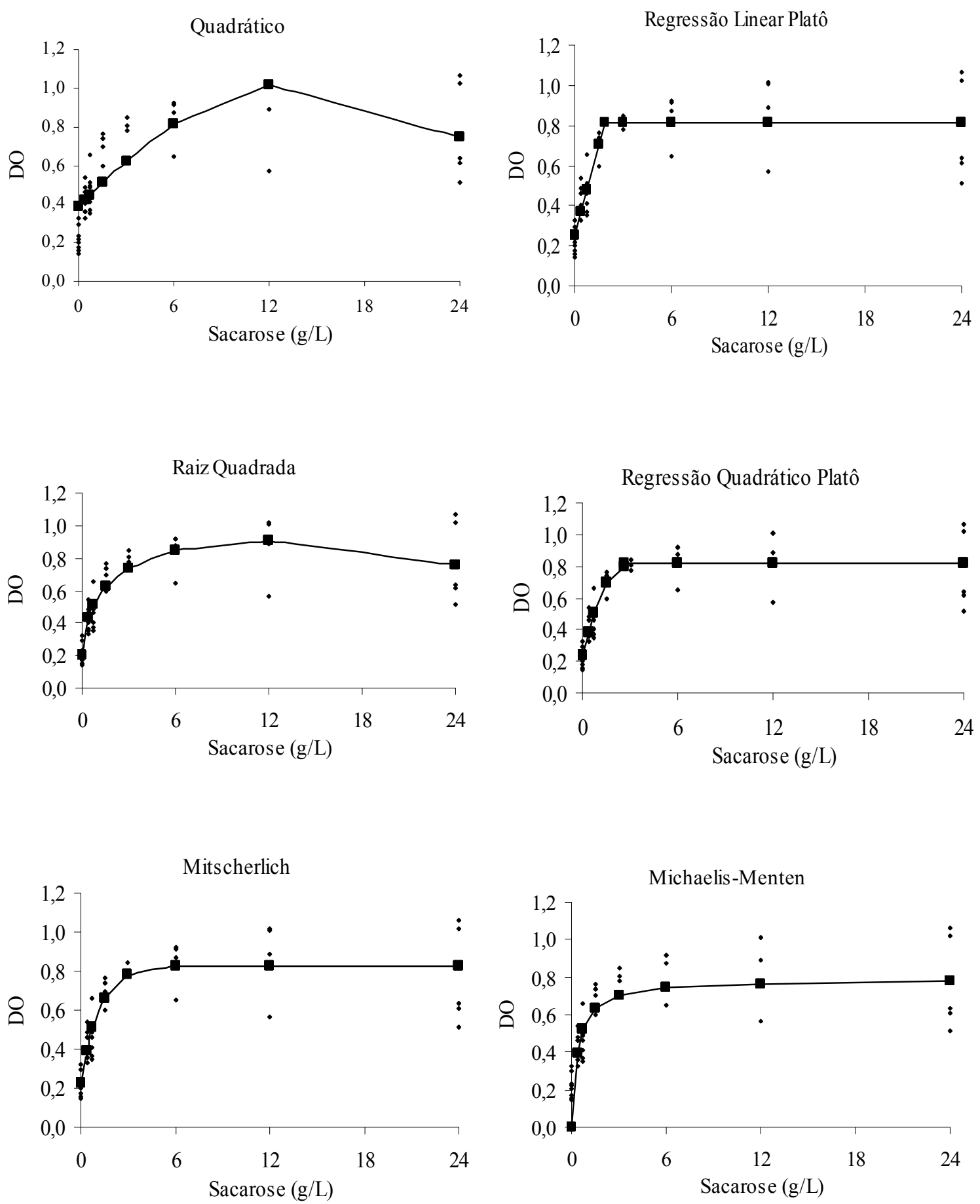

Figura 1. Crescimento microbiano ruminal (DO $600 \mathrm{~nm}$ ) em função da concentração de sacarose $(\mathrm{g} / \mathrm{L})$ segundo diversos modelos. 
Rev. Bras. Saúde Prod. Anim., Salvador, v.13, n.2, p....-... abr./jun., 2012 http://www.rbspa.ufba.br ISSN 15199940

Tabela 3. Médias, estimativas de parâmetros de regressão e nível de probabilidade para o erro tipo I associado à hipótese de nulidade conjunta, entre a relação funcional dos valores de crescimento microbiano medido pela densidade ótica (DO $-600 \mathrm{~nm}$ ) observados e aqueles preditos pelos modelos analisados

\begin{tabular}{lccccc}
\hline \multirow{2}{*}{ Modelo } & \multirow{2}{*}{$\begin{array}{c}\text { Média DO }(600 \mathrm{~nm}) \\
\text { Predito (X) }\end{array}$} & $\beta_{o}$ & $B_{1}$ & $\mathrm{R}^{2}$ & Valor-P $^{1}$ \\
\hline Linear-Platô & 0,627 & $-0,0368$ & 1,035 & 0,77 & 0,7694 \\
Quadrático & 0,615 & 0,0447 & 0,9230 & 0,58 & 0,8684 \\
Quadrático Platô & 0,627 & $-0,0410$ & 1,042 & 0,78 & 0,7422 \\
Raiz quadrada & 0,625 & $-0,0133$ & 1,002 & 0,75 & 0,8705 \\
Mitscherlich & 0,668 & $-0,0400$ & 1,045 & 0,79 & 0,7734 \\
Michaelis-Menten & 0,645 & $-0,0740$ & 1,093 & 0,67 & 0,7498 \\
\hline Valor observado (Y) & 0,612 & - & - & - & -
\end{tabular}

${ }^{\mathrm{T}} H \mathrm{o}: \beta \mathrm{o}=0 \mathrm{e} \beta_{1}=1$.

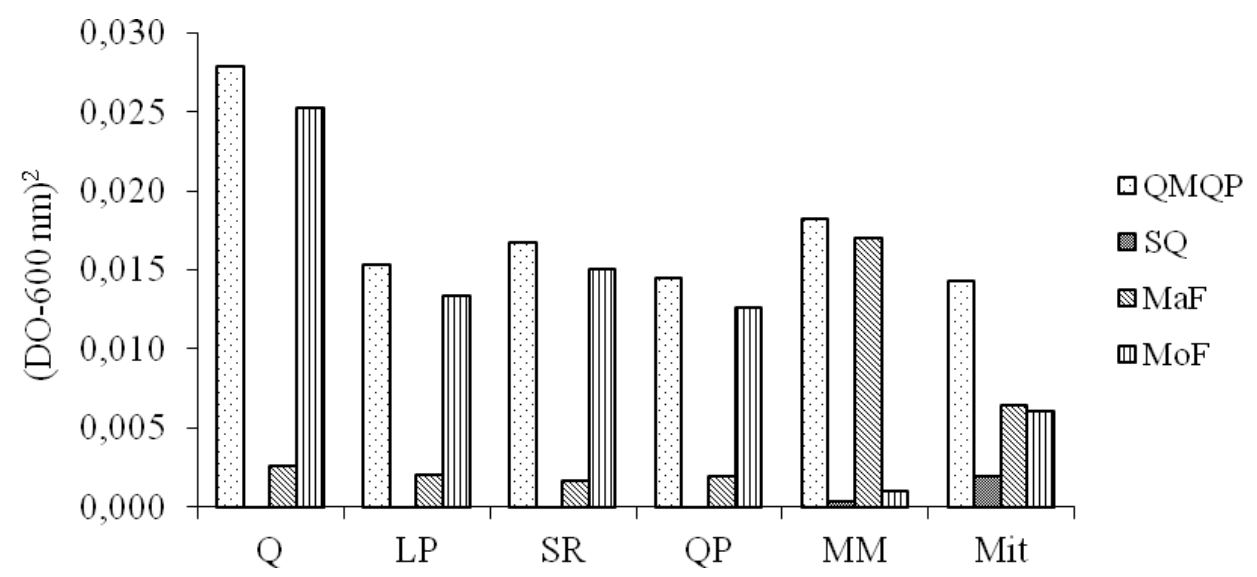

Figura 2. Partição do quadrado médio do erro de predição (QMEP) para densidade óptica (DO - 600nm) nos componentes quadrado do vício total $(\mathrm{QV})$, desvio na magnitude de flutuação $(\mathrm{MaF})$ e desvio no modelo de flutuação (MoF) para os modelos quadrático (Q), linear-platô (LP), raiz quadrada (RQ), quadrático platô (QP), Michaelis-Menten (MM) e Mitscherlich (Mit)

Os modelos linear-platô, raiz quadrada e quadrático platô apresentam qualidades de predição semelhantes. Os mesmos, além de gerarem estimativas nãoviesadas de crescimento microbiano (baixo QV), simulam o mesmo impacto (baixo MaF). Todavia, apresentam menor qualidade na direção da predição (maior participação do $\mathrm{MoF}$ ) em relação aos demais modelos (exceto o modelo quadrático), conforme visualização da dispersão dos dados acima e abaixo da reta de equalidade $(\mathrm{Y}=\mathrm{X})$ (Figura 3$)$. 
Rev. Bras. Saúde Prod. Anim., Salvador, v.13, n.2, p....-... abr./jun., 2012 http://www.rbspa.ufba.br ISSN 15199940

Quadrático

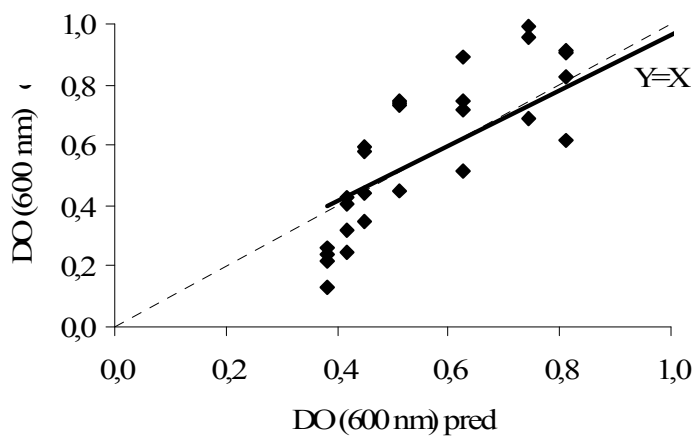

Quadrádito Platô

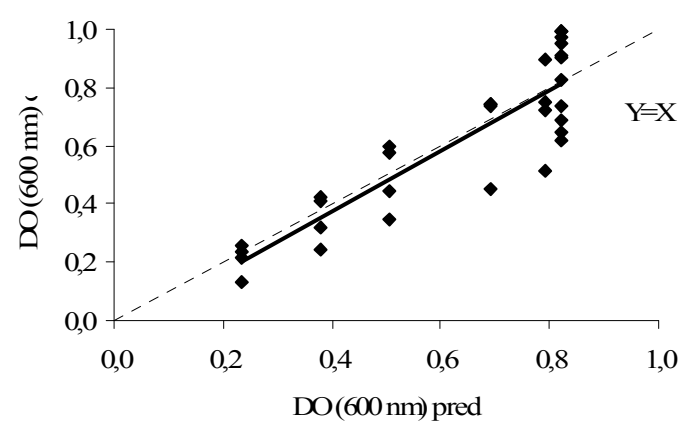

Mitscherlich

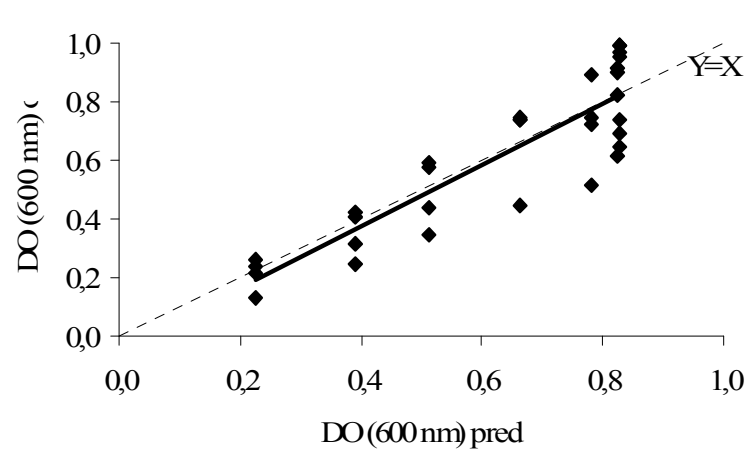

Linear Platô

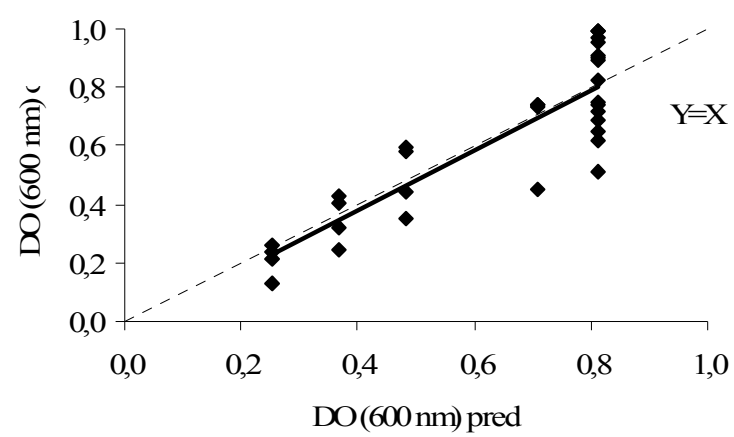

RaizQuadrada

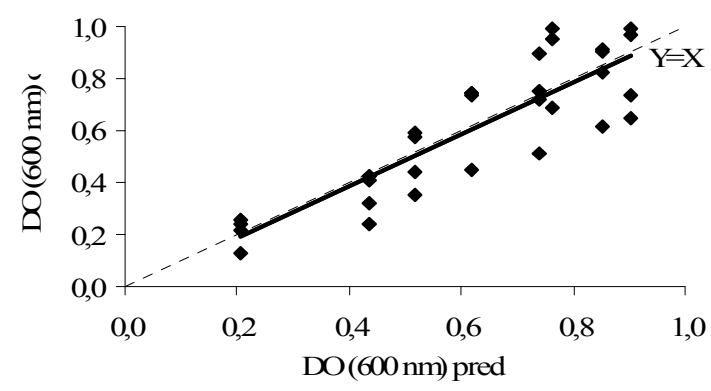

Michaelis-Menten

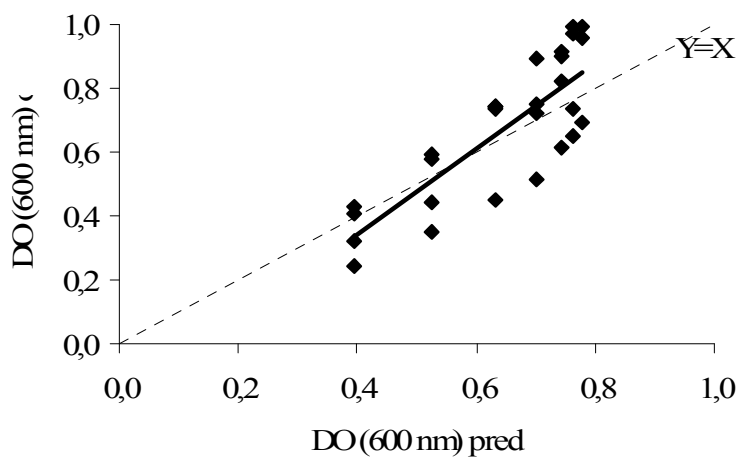

Figura 3. Relação entre os valores de crescimento microbiano, expresso pela densidade óptica (DO - $600 \mathrm{~nm}$ ) observados e preditos modelos. A linha contínua corresponde à reta dos mínimos quadrados.

Os modelos de Michaelis-Menten e Mitscherlich apresentaram comportamentos diferentes na qualidade de predição em relação aos demais.
Embora os valores de QMEP sejam semelhantes aos demais (exceção ao modelo quadrático), observaram-se diferenças principalmente na participação 
Rev. Bras. Saúde Prod. Anim., Salvador, v.13, n.2, p....-... abr./jun., 2012 http://www.rbspa.ufba.br ISSN 15199940

do MaF e Mof. O modelo de MichaelisMenten apresentou falhas na simulação do mesmo impacto (alto MaF), mas gera estimativas não-viesadas de crescimento microbiano (baixo QV) e apresenta melhor qualidade na direção da predição (baixo Mof) entre todos os modelos. No modelo Mitscherlich, embora gere estimativas com maior vício de predição entre os modelos (QV), apresenta boa qualidade na simulação do mesmo impacto $(\mathrm{MaF}) \mathrm{e}$ na direção da predição (MoF) (Figura 3).

Cerrato \& Blackmer (1990) ao comparar cinco de modelos (quadrático, linear-platô, quadrático-platô, raiz quadrada e Mitscherlich) para descrever respostas produtivas e econômicas da produção de milho grão à fertilização nitrogenada, concluíram que o modelo quadrático-platô é o que melhor descreve o comportamento das respostas. Todavia, para avaliação dos modelos, os autores utilizaram análise de resíduos com o mesmo banco de dados para construção dos modelos, o que viola os princípios da avaliação de qualidade da predição de modelos (TEDESCHI, 2006).

Com exceção ao modelo quadrático, a partição do QMEP não permitiu identificar diferenças marcantes em relação à qualidade na predição dos modelos. Discussões sobre as estimativas de indicadores de eficiência de utilização dos nutrientes poderão melhor discriminar as diferenças na qualidade da predição (Tabela 4). Neste sentido, os valores estimados de crescimento microbiano máximo (DOmax) apresentaram baixa variação entre os modelos (DO - 600nm entre 1,03 a 0,7882). Porém, observa-se elevada variação nas estimativas da concentração de sacarose que permite $50 \%$ do crescimento microbiano máximo $\left(\mathrm{SAC}_{50 \% \mathrm{MAX}}, \mathrm{g} / \mathrm{L}\right)$ e da concentração de sacarose que maximiza o crescimento microbiano ( $\left.\mathrm{SAC}_{\mathrm{MAX}}\right)$.

Tabela 4. Equação de predição do crescimento microbiano marginal (CMa) em função da concentração de sacarose ( $\mathrm{SAC}, \mathrm{g} / \mathrm{L})$, concentração de sacarose que permite $50 \%$ do crescimento microbiano máximo $\left(\mathrm{SAC}_{50 \% \mathrm{MAX}}, \mathrm{g} / \mathrm{L}\right)$, crescimento microbiano máximo (DO $\mathrm{MAX}_{\mathrm{M}}$ ) e concentração de sacarose no ponto de $\mathrm{DO}_{\mathrm{MAX}}$, estimados segundo vários modelos

\begin{tabular}{|c|c|c|c|c|c|}
\hline \multirow[b]{2}{*}{ Modelo $^{1}$} & \multirow{2}{*}{$\begin{array}{l}\text { Crescimento marginal (CMa) } \\
\text { (D.O./g SAC) }\end{array}$} & \multicolumn{2}{|c|}{$\mathrm{SAC}_{50 \% \mathrm{MAX}}$} & \multirow{2}{*}{$\begin{array}{l}\text { DOmax } \\
(600 \mathrm{~nm})\end{array}$} & \multirow{2}{*}{$\begin{array}{l}\text { SACmax } \\
\quad(\mathrm{g} / \mathrm{L})\end{array}$} \\
\hline & & $(g / L)$ & $\begin{array}{c}\% \\
\text { SACmax }\end{array}$ & & \\
\hline Q & $0,0902-0,00626 * \mathrm{SAC}$ & 1,573 & $10,9 \%$ & 1,03 & 14,41 \\
\hline LP & $-0,302, \mathrm{SAC}<1,8482 ; 0, \mathrm{SAC} \geq 1,8482$ & 0,504 & $27,3 \%$ & 0,8133 & 1,848 \\
\hline QP & $\begin{array}{l}0,4244-0,159 * \mathrm{SAC}, \mathrm{SAC}<2,67 ; 0, \mathrm{SAC} \\
\geq 2,67\end{array}$ & 0,458 & $17,2 \%$ & 0,8209 & 2,67 \\
\hline RQ & $-0,0613+0,2068 * \mathrm{SAC}^{-0,5}$ & 0,4350 & $3,8 \%$ & 0,904 & 11,38 \\
\hline M & $0,8262 * \exp ^{-0,8713 *(\mathrm{SAC}+0,3631)}$ & 0,4314 & $5,7 \%$ & 0,8254 & 7,60 \\
\hline MM & $0,2962 /\left(0,1412+0,756 \cdot \mathrm{SAC}+\mathrm{SAC}^{2}\right)$ & 0,3758 & $5,3 \%$ & 0,7882 & $7,14^{2}$ \\
\hline
\end{tabular}


O modelo raiz quadrada e MichaelisMenten estimaram valores mais próximos de $\mathrm{SAC}_{50 \% \mathrm{MAX}}$, expresso em g/L. No entanto, como o valor do SAC $_{\text {MAX }}$ estimado pelo modelo raiz quadrada foi muito maior $(83,8 \%) \mathrm{em}$ relação ao modelo Michaelis-Menten, o valor de $\mathrm{SAC}_{50 \% \mathrm{MAX}}$, expresso em \% $\mathrm{SAC}_{\mathrm{MAX}}$, evidentemente, foi menor. Os modelos de Mitscherlich e MichaelisMenten estimaram de forma mais semelhante todos os valores de eficiência de utilização de nutrientes.

A diferença nas estimativas de crescimento marginal entre os modelos apresenta importante implicação prática, pois a função de produtividade marginal tem sido utilizada para predição do nível ótimo econômico de nutrientes em sistemas de produção agrícola e animal (CERRATO \& BLACKMER, 1990; OLIVEIRA et al., 2010). Segundo Oliveira et al. (2010), a resposta produtiva marginal do suplemento de nutrientes e a relação de preços entre suplemento e o produto (ex. leite) são as variáveis chaves que definem o nível ótimo de suplementação para bovinos de leite. Neste sentido, os níveis ótimos de suprimento de nutrientes podem variar com o modelo utilizado.

$\mathrm{O}$ modelo quadrático subestima e eficiência de utilização de nutrientes (crescimento microbiano marginal-CMa em função da concentração de sacarose) nos níveis mais baixos de sacarose $(<2 \mathrm{~g} / \mathrm{L})$ e superestima nos níveis mais altos, em relação aos demais modelos. O modelo quadrático-platô, embora estime maiores valores de eficiência nos níveis mais baixos de sacarose em relação ao modelo quadrático, o comportamento linear decrescente do CMa até o nível 0 (como no platô DO é constante, $\mathrm{CMa}=0$ ), não condiz adequadamente com o comportamento curvilíneo dos sistemas biológicos (Figura 4).

Embora as ferramentas estatísticas de avaliação de modelos indiquem adequada qualidade na predição do crescimento microbiano do modelo linear-platô, a visualização do comportamento do crescimento microbiano marginal demonstra inadequação biológica. Reconhecidamente, os sistemas biológicos apresentam rendimentos decrescentes com aumento no suprimento de nutrientes (LANA et al., 2007). No entanto, segundo este modelo, o CMa é constante até atingir o máximo crescimento (platô), a partir do qual o CMa torna-se nulo.

O CMa estimados pelos modelos raiz quadrada, Mitscherlich e MichaelisMenten apresentam-se mais adequados, principalmente nos níveis mais baixos de sacarose. Nesses modelos, o CMa decresce de maneira exponencial, com redução mais drástica nos níveis mais baixos de sacarose. No entanto, a partir de um determinado nível de sacarose, a redução no CMa é minimizada e atinge estabilização acima do eixo de $\mathrm{X}$ (exceto 0 modelo raiz quadrada), próxima ao nível zero de CMa. Este comportamento observado nos maiores níveis de substratos, aparentemente, é menos condizente com as respostas de sistemas biológicos ao suprimento de nutrientes em sistemas fechados, que apresentam fase de declíneo e morte celular por saturação enzimática e/ou por excesso de produtos do metabolismo substrato, em condições de excesso de substratos. Nesta situação esperar-se-ia $\mathrm{CMa}$ negativos, somente verificado nos modelos quadráticos e raiz quadrada.

Por outro lado, espera-se que o nível ótimo de nutrientes seja recomendado em níveis inferiores ao nível em que se inicia o processo de intoxicação. Portanto, modelos de saturação cinética, 
Rev. Bras. Saúde Prod. Anim., Salvador, v.13, n.2, p....-... abr./jun., 2012 http://www.rbspa.ufba.br ISSN 15199940

como raiz quadrada, Mitscherlich e Michaelis-Menten parecem ser os mais adequados sob os aspectos biológicos, econômicos e ambientais.

Como os sistemas microbiológicos apresentam comportamento ao suprimento de nutrientes semelhantes aos sistemas macrobiológicos vegetal e animal, os mesmos podem ser utilizados para descrever o comportamento da
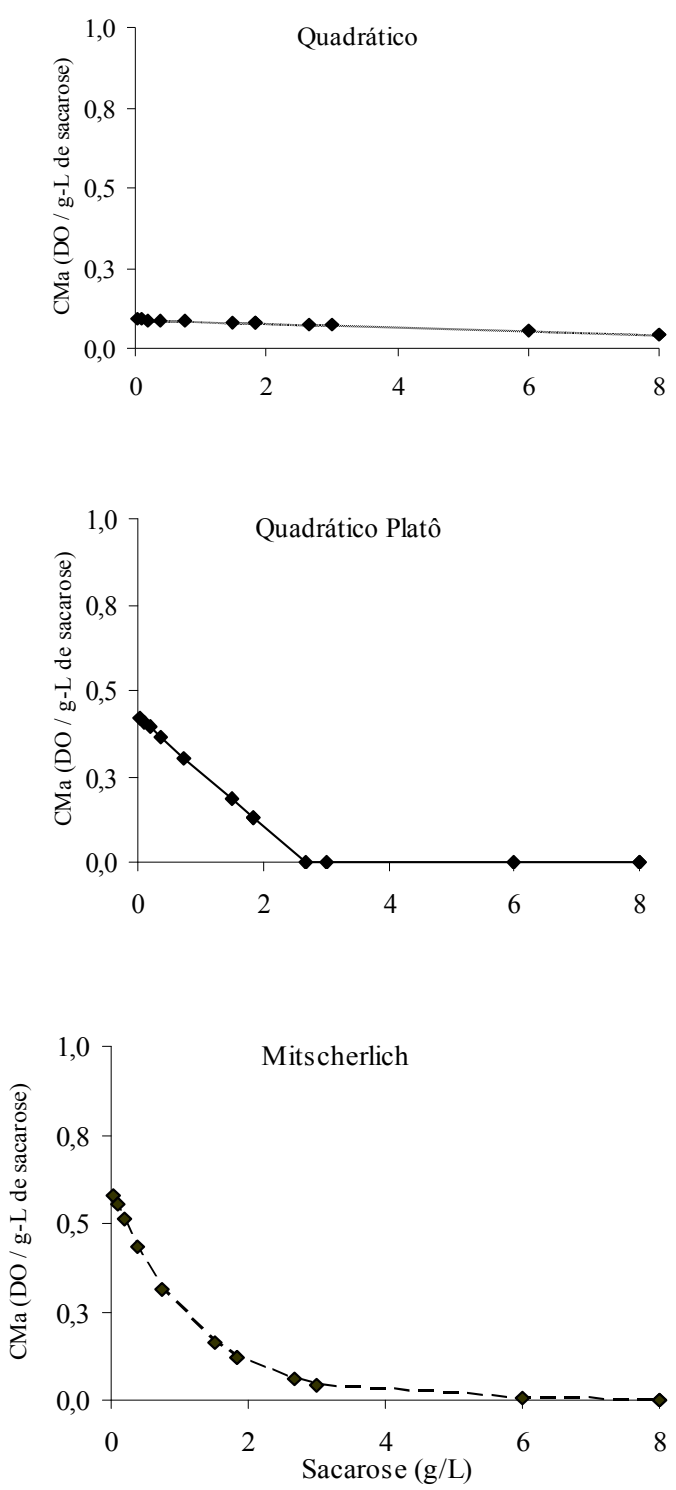

maioria dos sistemas biológicos de interesse econômico (LANA et al., 2005; LANA et al., 2007; OLIVEIRA et al., 2010). Neste sentido, as diferenças na predição do $\mathrm{CMa}$ ao suprimento de nutrientes entre os modelos apresentam importantes implicações práticas para a maioria dos sistemas de produção vegetal e animal de interesse econômico.
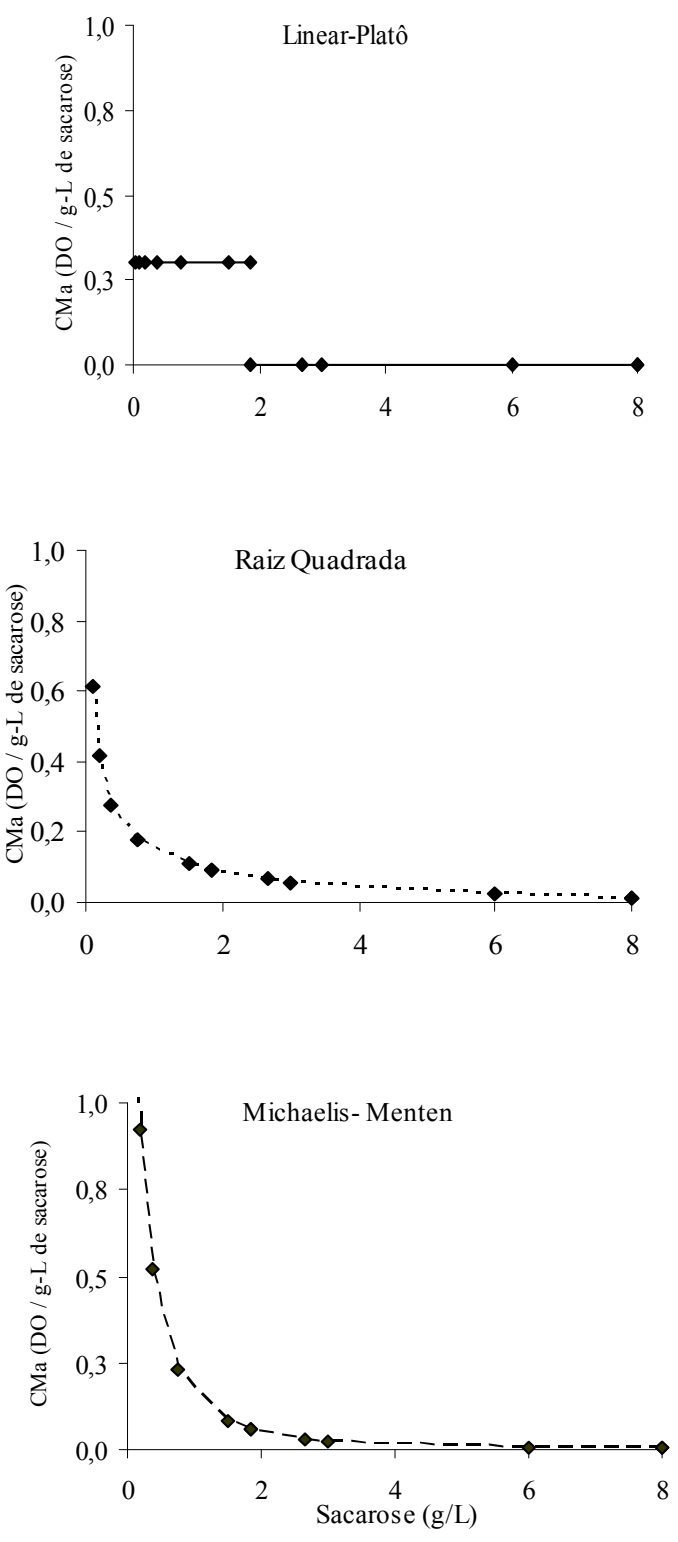

Figura 4. Crescimento microbiano ruminal marginal (CMa) em função da concentração de de sacarose $(\mathrm{g} / \mathrm{L})$ estimados por diversos modelos 
Rev. Bras. Saúde Prod. Anim., Salvador, v.13, n.2, p.457-468 abr./jun., 2012 http://www.rbspa.ufba.br ISSN 15199940

O modelo linear-platô mostra-se inadequado para descrever $\mathrm{o}$ comportamento do crescimento microbiano ruminal ao suprimento de nutrientes, da mesma forma que $o$ modelo quadrático quando em baixos níveis de suprimento de nutrientes.

Os modelos quadrático-platô, raiz quadrada, Mitscherlich ou MichaelisMenten podem ser utilizados para descrever o comportamento do crescimento microbiano ruminal ao suprimento de nutrientes. Porém, diferenças são observadas quanto às estimativas das eficiências de uso nos diferentes níveis de substratos, o que implica em diferenças nas recomendações dos níveis ótimos de nutrientes. Neste sentido, a escolha desses modelos poderá ser modulada pelo nível de suprimento de nutrientes desejado.

\section{REFERÊNCIAS}

CERRATO, M.E.; BLACKMER, A.M. Comparasion of models for describing corn yield response to nitrogen fertilizer. Agronomy Journal, v.82, p.138-143, 1990.

KOBAYASHI, K.; SALAM, M.U. Comparing simulated and measured values using mean squared deviation and its components. Agronomy Journal, v.92, n.2, p.345-352, 2000.

LANA, R.P.; FALEIRO NETO, J.A.; PAIVA, V.C.R. Crescimento de bactérias ruminais em função da concentração de substratos energéticos e nitrogenado no meio de cultura. In: LANA, R.P. (Ed.) Respostas biológicas aos nutrientes. Viçosa, MG: Universidade Federal de Viçosa, 2007. p.87-102.
LANA, R.P.; GOES, R.H.T.B.; MOREIRA, L.M.; MÂNCIO, A.B.; FONSECA, D.M.; TEDESCHI, L.O. Application of lineweaver-Burk data transformation to explain animal and plant performance as a function of nutrient supply. Livestock Production Science, v.98, n.3, p.219-224, 2005.

LOPEZ, S.; PRIETO, M.; DIJKSTRA, J.; DHANOA, M.S.; FRANCE, J. Statistical evaluation of mathematical models for microbial growth. International Journal of Food Microbiology, v.96, p.289-300 2004.

MAYER, D.G.; STUART, M.A.; SWAIN, A.J. Regression of real-world data model output: an appropriate overall test of validity. Agriculture Systems, v.45, p.93-104, 1994.

MESQUITA, E.E; NERES, M.A. Morfogênese e composição bromatológica de cultivares de Panicum maximum em função da adubação nitrogenada. Revista Brasileira de Saúde e Produção Animal [Online], v.9, n.2, p. 201-209, 2008.

OLIVEIRA, A.S.; CAMPOS, J.M.S.; LANA, R.P.; DETMANN, E.; VALADARES FILHO, S.C. Estimation of optimal level of concentrates for dairy cows on tropical pastures by marginal analysis. Revista Brasileira de Zootecnia, v.39, p.2040-2047, 2010.

POUILLOT, R.; LUBRAN, M.B. Predictive microbiology models vs. modeling microbial growth within Listeria monocytogenes risk assessment: what parameters matter and why. Food Microbiology, v.28, p.720-726, 2011.

TEDESCHI, L.O. Assessment of the adequacy of mathematical models. Agricultural Systems, v.89, p.225-247, 2006.

Data de recebimento: 21/03/2011

Data de aprovação: 24/04/2012 